\title{
BMJ Open Design of the HEM-POWR study: a prospective, observational study of real- world treatment with damoctocog alfa pegol in patients with haemophilia $A$
}

\author{
Martin Sanabria, ${ }^{1}$ María Teresa Álvarez Román, ${ }^{2}$ Giancarlo Castaman, ${ }^{3}$ \\ Maissaa Janbain, ${ }^{4}$ Tadashi Matsushita, ${ }^{5}$ Karina Meijer (i) , ${ }^{6}$ Johannes Oldenburg, ${ }^{7}$ \\ Sabine Friedl, ${ }^{8}$ M T Reding (iD ${ }^{9}$
}

To cite: Sanabria M, Álvarez Román MT, Castaman G, et al. Design of the HEM-POWR study: a prospective, observational study of real-world treatment with damoctocog alfa pegol in patients with haemophilia A. BMJ Open 2021;11:e044997. doi:10.1136/ bmjopen-2020-044997

- Prepublication history and additional supplemental material for this paper are available online. To view these files, please visit the journal online (http://dx.doi.org/10.1136/ bmjopen-2020-044997).

Received 18 September 2020 Accepted 04 August 2021

Check for updates

(C) Author(s) (or their employer(s)) 2021. Re-use permitted under CC BY-NC. No commercial re-use. See rights and permissions. Published by BMJ.

For numbered affiliations see end of article.

Correspondence to

Dr M T Reding;

redin002@umn.edu

\section{ABSTRACT}

Introduction Haemophilia A is a rare bleeding disorder caused by defects in coagulation factor VIII (FVIII). Damoctocog alfa pegol (BAY 94-9027, Jivi, Bayer, Germany) is a site-specifically PEGylated, extended-halflife, recombinant FVIII, approved for use in previously treated patients (PTPs) aged $\geq 12$ years with haemophilia A. However, a real-world evidence regarding routine clinical use of damoctocog alfa pegol is limited.

Methods and analysis HEM-POWR is a multinational, multicentre, non-interventional, prospective, postmarketing cohort study evaluating the effectiveness and safety of real-world treatment with damoctocog alfa pegol. Estimated enrolment is $\geq 200$ PTPs with haemophilia A, receiving damoctocog alfa pegol (on-demand, prophylaxis or intermittent prophylaxis (as per local label)), observed for 36 months. Primary outcomes are total bleeding events and annualised bleeding rate; secondary outcomes include long-term safety, joint health, pharmacokinetics, patientreported outcomes (PROs) from validated questionnaires and perioperative haemostasis. Where applicable, reasons for switching to damoctocog alfa pegol, choice of treatment regimen and dose will also be captured. Exploratory and descriptive statistical analyses will be performed, and will be stratified by parameters including, but not limited to, prophylaxis regimen and haemophilia severity. Patients can record bleeds and consumption in electronic (e) Diaries, ePROs, and can access nonpromotional study information (videos explaining study procedures) via an online patient portal. Optionally, patients can enrol in the LIFE-ACTIVE substudy designed to investigate the relationship between activity (measured by the ActiGraph CP Insight watch) and effectiveness parameters collected from HEM-POWR.

Ethics and dissemination Study approval was obtained by local independent ethics committees and authorities in participating study centres across Europe, the Americas and Asia. Informed consent from patients or their legal representative is a requirement for participation. The study results will be submitted for publication in a peerreviewed scientific journal and presented at scientific conferences.

Trial registration numbers NCT03932201, EUPAS26416. Protocol version and date V.1.2, 27 September 2019.

\section{Strengths and limitations of this study}

- This is the first study to provide prospective data on the effectiveness, utilisation and long-term safety of damoctocog alfa pegol in patients with haemophilia $A$ in routine clinical practice.

- The prospective, observational design allows the study to capture prespecified real-life data.

- The use of an online patient portal may facilitate patient retention over the study.

- The risk of bias due to the non-interventional design is a limitation.

\section{INTRODUCTION}

Haemophilia A is an X-linked, congenital bleeding disorder caused by defects in coagulation factor VIII (FVIII) and has an estimated prevalence of one in 10000 births. ${ }^{1}$ Patients with haemophilia experience spontaneous bleeding into joints or muscles. ${ }^{1}$ Patients with severe haemophilia, defined as having $<1 \mathrm{IU} / \mathrm{dL}(<1 \%$ of normal) endogenous FVIII activity, may suffer from recurrent joint bleeds that could lead to haemophilic arthropathy and progressive disability. ${ }^{1}$

The standard of care for patients with haemophilia $\mathrm{A}$ is prophylactic replacement of FVIII using either plasma derived or recombinant (r)FVIII. ${ }^{12}$ Prophylaxis with FVIII is recommended for patients with severe or moderate haemophilia A who have a relatively high bleeding rate and currently includes the use of standard-half-life (SHL) and extended-half-life (EHL) FVIII products. Prophylaxis with SHL rFVIII products requires intravenous infusions between three and four times per week, which can lead to suboptimal adherence and breakthrough bleeding. ${ }^{34}$ EHL products, on the other hand, offer longer intervals between infusions (one 
to three times per week) while still meeting efficacy and safety outcomes. ${ }^{5}$

Damoctocog alfa pegol (BAY 94-9027, Jivi, Bayer, Germany) is a B-domain-deleted EHL rFVIII product that has been site-specifically conjugated with polyethylene glycol (PEGylated) to extend its half-life. ${ }^{6}$ In previously treated patients (PTPs) with severe haemophilia A, damoctocog alfa pegol demonstrated a 1.4-fold increase in half-life and dose-normalised area under the curve (AUC) compared with an SHL rFVIII product (sucroseformulated rFVIII (Kogenate FS, Bayer, Berkeley, California, USA) $).{ }^{78}$ Furthermore, damoctocog alfa pegol demonstrated improvements in AUC and half-life compared with another EHL rFVIII product, rFVIII Fc fusion protein (Elocta, Swedish Orphan Biovitrum, UK), following a single infusion in patients with severe haemophilia A. ${ }^{9}$ A study comparing damoctocog alfa pegol against another EHL rFVIII product, rurioctocog alfa pegol (Adynovate/Adynovi, Takeda, Japan) has been submitted for publication. ${ }^{10}$

In the phase 2/3 PROTECT VIII study, damoctocog alfa pegol prevented bleeding in previously treated adults and adolescents with severe haemophilia A at dosing intervals of up to every 7 days, and effectively treated bleeds when used on demand. ${ }^{5}$ Damoctocog alfa pegol was well tolerated and no patients developed inhibitors. ${ }^{5}$ Furthermore, damoctocog alfa pegol was efficacious and well tolerated in the treatment of patients undergoing surgeries. ${ }^{11}$

Based on these results, damoctocog alfa pegol was approved for prophylactic, on-demand and perioperative management of bleeding in PTPs aged $\geq 12$ years with haemophilia A by the US Food and Drug Administration, the European Medicines Agency, the Pharmaceuticals and Medical Devices Agency in Japan and Health Canada in 2018. Interim data from the PROTECT VIII extension study confirm long-term safety of damoctocog alfa pegol, and maintenance of a low annualised bleeding rate $(\mathrm{ABR})$ with extended-interval prophylactic dosing in patients with severe haemophilia A. ${ }^{12}$

Although clinical trial data offer robust, controlled analyses for large cohorts of patients, they may not always be reflective of real-world clinical practice; therefore, it is important to determine whether the efficacy and safety observed during the PROTECT VIII study extends to the real world. The purpose of this manuscript is to describe the design of the HEM-POWR study, an ongoing postmarketing study evaluating the effectiveness and safety of real-world treatment with damoctocog alfa pegol in PTPs with haemophilia A.

\section{METHODS}

\section{Study design}

HEM-POWR is a multinational, multicentre, noninterventional, prospective cohort study including participating study centres across Europe, the Americas and Asia. The primary study objective is to assess the effectiveness of damoctocog alfa pegol prophylaxis in a real-world setting through collection of total bleeding events and analysis of ABR. The study aims to enrol $\geq 200$ patients with haemophilia A, including both patients who have not yet been treated previously with damoctocog alfa pegol (but for whom the decision to receive treatment with damoctocog alfa pegol has already been made) and patients who are currently receiving damoctocog alfa pegol (on-demand, prophylaxis or intermittent prophylaxis) at study centres throughout Europe, the Americas and Asia. Patients and physicians can choose from different prophylaxis regimens with damoctocog alfa pegol following approved local labels, or any regimen prescribed by the physician as part of normal clinical practice. The dosing regimen can be changed based on the patient's response to treatment at any time and the reason for the change will be documented.

Patients can also register for the online study-specific patient portal that provides access to the electronic bleed diary (eDiary) and electronic quality of life questionnaires (electronic patient-reported outcomes, ePROs) in the electronic data capture (EDC) system. The portal also contains non-promotional study information.

Due to the observational nature of the study, no specific follow-up visits are scheduled. Data collection will occur continuously throughout the 3-year observational period and will be documented in the electronic case report form (eCRF) contained in the EDC system. The last safety follow-up will be completed up to 30 days after receiving the final damoctocog alfa pegol treatment within the study period. Final data collection point will not happen sooner than 36 months after the initial visit, and thus, the data documentation period will continue for up to 3 months after month 36 from the final follow-up time point. The study could potentially be extended beyond the 3-year period, subject to the study initiator's decision.

\section{Study population}

To minimise bias in the selection of patients, investigators at participating sites are encouraged to enrol all eligible patients receiving damoctocog alfa pegol. The study will employ a limited number of inclusion and exclusion criteria to reflect the diversity of patients encountered in clinical practice (table 1 ).

\section{HEM-POWR study assessments}

The primary endpoint is the mean/median annualised number of reported total bleeds in patients with haemophilia A (figure 1). Secondary endpoints include longterm safety outcomes, the annualised number of joint, spontaneous and trauma bleeds, pharmacokinetics, patient-reported quality of life outcomes and proportion of patients with zero bleeds (see box 1 for full list of secondary endpoints).

Baseline demographic and medical history data will be collected at study enrolment by retrospective chart review and will include lifetime duration on prophylaxis, data on previous therapy, pharmacokinetic parameters, medical 
Table 1 Inclusion and exclusion criteria

\begin{tabular}{ll}
\hline Inclusion criteria & Exclusion criteria \\
\hline Diagnosis of haemophilia A & $\begin{array}{l}\text { Concurrent participation } \\
\text { Patients previously treated }\end{array}$ \\
in an investigational \\
programme with \\
Patients without previous & $\begin{array}{l}\text { interventions outside of } \\
\text { history of FVIII inhibitors }\end{array}$ \\
or patients with previous & Diagnosis of any other \\
history of FVIII inhibitors on & bleeding/coagulation \\
standard prophylaxis therapy & disorder other than \\
for $\geq 1$ year prior to study & haemophilia A \\
entry & Contraindications \\
No current evidence, ${ }^{*}$ or & according to the local \\
clinical suspicion† of FVIII & marketing authorisation \\
inhibitor & Receiving ITI treatment \\
Initiation of, or currently & at enrolment \\
on, damoctocog alfa pegol & \\
with any kind of treatment & \\
modality (on-demand, & \\
prophylaxis or intermittent & \\
prophylaxis) & \\
Signed informed consent/ & \\
assent & \\
\hline
\end{tabular}

${ }^{*}$ As measured by the Nijmegen-modified Bethesda assay $(<0.6$ Bethesda units $(\mathrm{BU}) / \mathrm{mL})$ or Bethesda assay $(<1.0 \mathrm{BU} / \mathrm{mL})$ on two consecutive samples.

†Documented or clinical suspicion of shortened FVIII half-life ( $<6$ hours).

FVIII, factor VIII; ITI, immune tolerance induction.

condition and bleeds up to 12 months prior to administration of damoctocog alfa pegol (table 2).

Patients will be advised to record all bleeding events and administered infusions, preferably in the eDiary, which can be accessed via the online patient portal. Alternatively, patients will be allowed to record their bleeding events in a paper diary, which will then be transferred to eCRF by the investigator. Details of treatments for surgery will be collated separately in the eCRF (table 2). Adverse events (AEs) will be documented in the AE Report Form (part of the eCRF) and reported to the marketing authorisation holder, according to local requirements.

\section{LIFE-ACTIVE substudy assessments}

Patients can enrol in the optional LIFE-ACTIVE substudy (except in Japan) to investigate the relationship between activity and effectiveness parameters collected from HEM-POWR (figure 1). Patients receiving damoctocog alfa pegol and willing to participate in the LIFE-ACTIVE substudy will be asked to wear an ActiGraph CP Insight activity-tracking smart watch continuously for 30 days from their initial visit and again following their follow-up visits approximately around months 12, 24 and 36 (visits are expected to coincide with the routine annual comprehensive haemophilia treatment centre appointments). Minutes per activity intensity will be recorded to determine levels of general mobility and physical activity intensity and duration. All data will be transferred to a secure, cloud-based system and patients will be blinded to values measured by the device.

\section{Statistical methods}

The study aims to enrol $\geq 200$ patients. Assuming a drop-out rate of $10 \%, 180$ patients will be available for the effectiveness analysis. Statistical analyses will be of an explorative and descriptive nature. The study is not designed to confirm or reject predefined hypotheses; hence, no formal hypothesis testing will be performed.

The analyses will be stratified based on prophylaxis regimen, severity of haemophilia, ABR at baseline, joint ABR at baseline, affected joints at baseline, patients' history of inhibitors and time on FVIII prior to treatment with damoctocog alfa pegol.

\section{Current status}

The first patient was enrolled in October 2019 and the study is expected to end in 2025 (last patient last visit

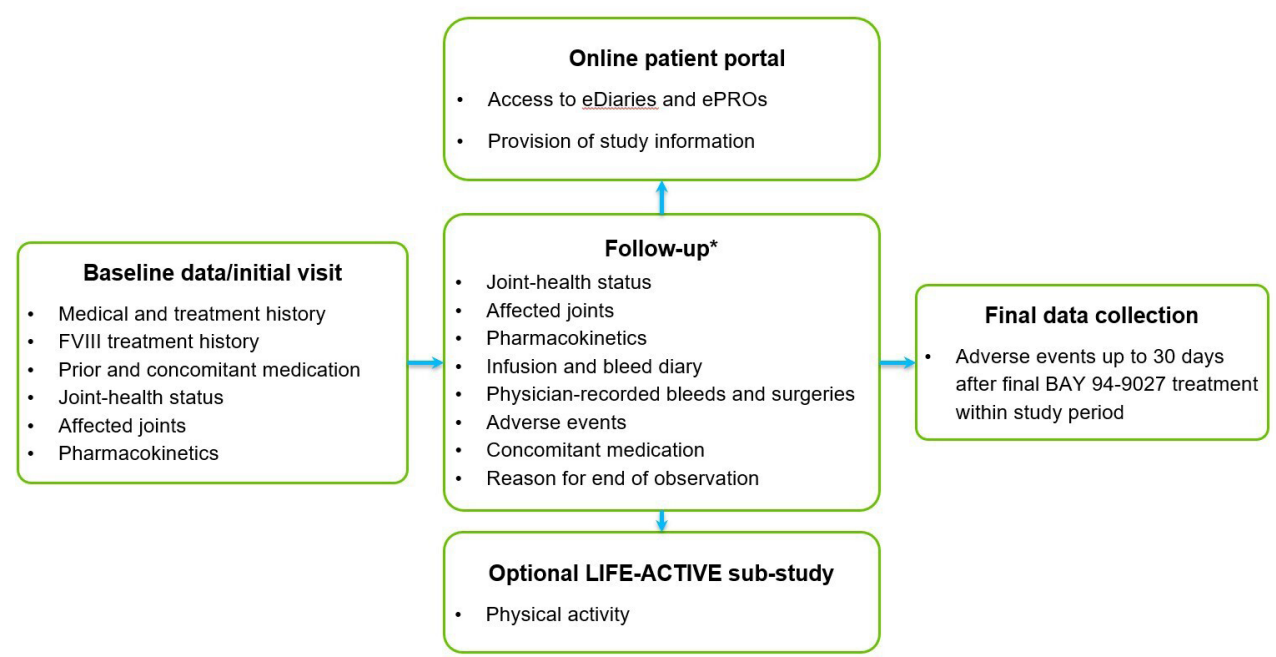

Figure 1 Outline of the HEM-POWR study including outcome measures *Until end of study ( $\geq 36$ months), premature discontinuation, withdrawal of consent or death. ePROs, electronic patient-reported outcomes; FVIII, factor VIII. 


\section{Box 1 HEM-POWR endpoints}

\section{Primary endpoint}

- Mean/median ABR at Months 12, 24 and 36.

\section{Secondary endpoints}

- Occurrence, duration, treatment, severity and outcome of AEs of special interest (hypersensitivity reactions, loss of drug effect, renal impairment, neurocognitive disorders and inhibitor development), AEs, SAEs and ARs.

- Number of infusions and FVIII consumption to achieve haemostasis during surgery.

- Change in HJHS from baseline to 12, 24 and 36 months.

- Joint status evaluation by ultrasound (HEAD-US score), if available, or part of standard clinical practice.

- Number of affected joints, ${ }^{*}$ and change from baseline at months 12,24 and 36.

- Annualised number of spontaneous, joint and trauma bleeds.

- Number of reported bleeds (total, spontaneous, joint and trauma) during the study compared with number of reported bleeds for previous FVIII products in the 12 months prior to enrolment into the study.

- Proportion of patients with no bleeds, and the difference in proportion compared with previous prophylaxis treatment.

- Description of pharmacokinetic parameters (half-life, FVIII trough, FVIII peak, IVR) for previous FVIII products versus damoctocog alfa pegol (if available in routine clinical practice).

- Effectiveness of treatment for break-through bleeds.

- Treatment satisfaction (PROs):

- Haemo-SAT ${ }_{A}$ score: change from baseline to 12,24 and 36 months.

- Haemo-QoL (A and SF) score: change from baseline to 12, 24 and 36 months.

- Work productivity and activity (PRO):

- WPAl score: change from baseline to 12, 24 and 36 months.

*Affected joint: a joint in which $\geq 3$ spontaneous bleeds have occurred within a consecutive 6-month period.

$A B R$, annualised bleeding rate; AEs, adverse events; ARs, adverse reactions; FVIII, factor VIII; Haemo-QoL (A and SF), haemophilia quality of life measure (for adults and short form for children); Haemo-SAT, haemophilia treatment satisfaction questionnaire for adults; HEAD-US, haemophilia early arthropathy detection with ultrasound; HJHS, Haemophilia Joint Health Score; IVR, in vivo recovery; PRO, patient-reported outcome; SAEs, serious adverse events; WPAl, work productivity and activity impairment.

expected in the second quarter of 2025), with yearly interim analyses.

\section{Ethics and dissemination}

In this observational study, data are collected based on routine clinical practice and patients are not believed to be exposed to further risks beyond regular therapy.

The physician will obtain informed consent from patients who are eligible for inclusion. The study results will be disseminated by presentation at scientific congresses and by publication in a peer-reviewed journal. Confidential data will be transferred in encoded form only. The entire documentation accessible to Bayer will not contain any data which, in conjunction with other freely available data or on its own account, could be used to identify specific persons. Personal patient records will not be made publicly available and will be kept confidential. Study findings stored electronically will be stored according to the local data protection laws. Availability of the data underlying this publication will be determined according to Bayer's commitment to the European Federation of Pharmaceutical Industries Association/Pharmaceutical Research and Manufacturers of America (EFPIA/ PhRMA), 'Principles for responsible clinical trial data sharing'. This pertains to scope, time point and process of data access. As such, Bayer commits to sharing, on request from qualified scientific and medical researchers, patientlevel clinical trial data, study-level clinical trial data and protocols from clinical trials in patients for medicines and indications approved in the USA and European Union (EU) as necessary for conducting legitimate research. This applies to data on new medicines and indications that have been approved by the EU and US regulatory agencies on or after 1 January 2014. Interested researchers can use www.clinicalstudydatarequest.com to request access to anonymised patient-level data and supporting documents from clinical studies to conduct further research that can help advance medical science or improve patient care. Information on the Bayer criteria for listing studies and other relevant information is provided in the study sponsors section of the portal.

For the full list of ethics committees that have approved of this study, please see online supplemental appendix 1 .

\section{Patient and public involvement}

Patients and the public were not involved in the design of this study. However, the research question and endpoints were designed so the results of which can be used to educate patients about real-world prophylaxis dosing regimens. In addition, patients, together with their physician, may choose from any of the locally approved BAY 94-9027 prophylaxis regimen that is most suitable for their needs and lifestyle.

\section{DISCUSSION}

HEM-POWR is the first study to provide prospective data on the effectiveness, utilisation and long-term safety of damoctocog alfa pegol in patients with haemophilia A in routine clinical practice. Postmarketing studies and registries are important for collecting real-world data (RWD) to assess the impact of treatments on broader patient populations than those included in controlled trials, and to monitor long-term safety and effectiveness.

RWD can be used across the healthcare community to back healthcare insurance coverage decisions and to develop guidelines and tools to support decision making in clinical practice. Characterising the patient experience of prophylaxis with damoctocog alfa pegol in a real-world setting will further validate and support current efficacy and safety data from previous clinical trials. ${ }^{511}$ Additionally, the reasons for changes in treatment type, dose and 
Table 2 Data collection during HEM-POWR

\begin{tabular}{lll}
\hline Eligibility assessment & $\times$ & $\times$ \\
Date & $\times$ & $\times$ \\
Demographic data & $\times$ & \\
General medical/surgical history & $\times$ \\
Disease history & $\times$ & \\
\hline Bleed history & $\times$ & $\times$ \\
\hline Prior FVIII treatment history & $\times$ & $\times$ (Continuous collection) \\
\hline Damoctocog alfa pegol history (if any) & $\times$ & \\
Current prescribed damoctocog alfa pegol regimen & & \\
Damoctocog alfa pegol dose/regimen changes/ & $\times$
\end{tabular}

treatment switches

\begin{tabular}{|c|c|c|}
\hline $\begin{array}{l}\text { PROs (Haemo-SAT }{ }_{A} \text {, Haemo-QoL-A, Haemo-QoL SF, } \\
\text { WPAI (+CIQ:HS)) }\end{array}$ & $\times$ & $\times($ Months $12,24,36)$ \\
\hline Physical examination (weight, height, vital signs)†,‡ & $\times$ & $\times$ \\
\hline Joint health (HJHS) & $\times$ & $\times$ \\
\hline Ultrasound data & & $\times$ \\
\hline Affected joints & & $\times$ \\
\hline Damoctocog alfa pegol PK analysis† & & $\times$ \\
\hline Concomitant medication & & $x$ \\
\hline $\begin{array}{l}\text { AEs (includes systematically collected AEs and AEs } \\
\text { of special interest)§ }\end{array}$ & $\times$ (Continuous collection) & \\
\hline Inhibitor† & $x$ & $x$ \\
\hline Patient infusion diary & & $\times($ Continuous collection) \\
\hline Physician recorded bleeds $\mathbb{1}$ & & $\times($ Continuous collection) \\
\hline Physician recorded surgeries & & $\times($ Continuous collection $)$ \\
\hline Reason for end of observation & & × (Once, at occurrence) \\
\hline
\end{tabular}

*Bleeding history will be recorded by the investigator, and will include number, type (spontaneous, trauma, joint, spontaneous joint and trauma joint), and location of all the bleeding events that the patient experienced within 12 months prior to starting therapy with damoctocog alfa pegol.

†Routine examinations only, if available.

$\ddagger$ PK analysis is at physician's discretion. PK testing (and the frequency of testing per the table) is part of the routine use of the product. $\S A E s$ (up to 30 days after the last treatment with damoctocog alfa pegol in study period).

ISerious, life-threatening bleeds and surgery bleeds to be recorded by the physician. Physician to ask patient at each visit regarding bleeds. AEs, adverse events; WPAI (+CIQ:HS), work productivity and activity impairment +classroom impairment questions: haemophilia specific; FVIII, factor VIII; Haemo-QoL (A and SF), haemophilia quality of life measure (for adults and short form for children); Haemo-SAT, haemophilia treatment satisfaction questionnaire for adults; HJHS, Haemophilia Joint Health Score; PK, pharmacokinetics; PROs, patient-reported outcomes.

dosing regimen collected in HEM-POWR will help to identify potential patterns.

HEM-POWR is designed to cover a broad range of locations (Europe, the Americas and Asia) and age range (adolescent, adult). Problems with enrolment, adherence and retention are common for observational studies; some online patient portals have been shown to improve patient adherence to medication, allow identification of medical errors, enhance the patient-provider communication and may improve clinical outcomes. ${ }^{13} 14$ The online patient portal used in HEM-POWR is designed to help improve patient adherence and engagement in the study by providing non-promotional study information and product-related information, as well as convenient access to the eDiary and ePROs.

The eligibility criteria have been designed to include a broad representation of patients with mild, moderate and severe haemophilia A within the study, and to minimise selection bias physicians will be asked to sample consecutive patients whenever possible.

When available, pharmacokinetic data from the HEMPOWR study could potentially be used to associate the improved pharmacokinetics of damoctocog alfa pegol with bleeding outcomes, relative to SHL FVIII treatments. ${ }^{8}$

Patients will also be invited to participate in LIFEACTIVE, a substudy to record patient activity levels in 
the context of effectiveness parameters collected during HEM-POWR, which could provide insights about physical activity in patients with haemophilia A.

The limitations of HEM-POWR include certain aspects of the study design to be expected of an observational study, such as lack of consistency in the format and schedule for recording patient data. Infusions and bleeding events are self-reported by patients and could introduce bias, and while the eDiary is a useful tool for study participants, its use is a recommendation rather than a necessity, and might not be used by all participants. As a result, a diverse set of diaries may be obtained with different information missing, varied level of detail and other inconsistencies which may be difficult to combine in one analysis. Another related limitation is the potential misclassification of bleeding events due to patients' self-reporting of outcomes, which is a common limitation of haemophilia observational studies. ${ }^{1516}$ This could be addressed through patient education programmes, which would train the patients to identify the different types of bleeding events accurately. Additionally, as the study is non-interventional, there is no established schedule for the follow-up visits; data will be collated approximately once a year at the patient's usual face-to-face or remote clinic visits during the 3-year observational period.

In conclusion, HEM-POWR is the first large-scale, observational study of damoctocog alfa pegol, which encompasses a broad range of locations and patient populations and uses an online patient portal to improve patient adherence and engagement. Together with data from the voluntary LIFE-ACTIVE substudy, the data will help us to understand the impact of physical activity on joint health in patients with haemophilia A. The results obtained are expected to support the efficacy and safety of damoctocog alfa pegol, observed in PROTECT VIII clinical trials, in a real-world setting, representing a step forward towards helping physicians and people with haemophilia A to achieve their desired treatment and life goals.

\author{
Author affiliations \\ ${ }^{1}$ Department of Hematology, Bayer AG, Basel, Switzerland \\ ${ }^{2}$ Hematology Department, Hospital Universitario La Paz, Madrid, Spain \\ ${ }^{3}$ Center for Bleeding Disorders and Coagulation, Department of Oncology, University \\ Hospital Careggi, Firenze, Italy \\ ${ }^{4}$ Center for Bleeding and Clotting Disorders, Tulane University School of Medicine, \\ New Orleans, Louisiana, USA \\ ${ }^{5}$ Department of Blood Transfusion Service, Nagoya University Hospital, Nagoya, \\ Japan \\ ${ }^{6}$ Faculty of Medical Sciences, University Medical Centre Groningen, Groningen, \\ Netherlands \\ ${ }^{7}$ Institute of Experimental Haematology and Transfusion Medicine, University \\ Hospital Bonn, Bonn, Germany \\ ${ }^{8}$ Department of Oncology and Heamatology, Bayer AG, Berlin, Germany \\ ${ }^{9}$ Center for Bleeding and Clotting Disorders, University of Minnesota Medical Center, \\ Minneapolis, Minnesota, USA
}

Acknowledgements Critical review and statistical support were provided by Inga Bayh, an employee of Bayer AG. Medical writing support was provided by Rebekka Harding-Smith and Frank Biegun of Darwin Healthcare Communications (Oxford and London, UK), funded by Bayer.
Contributors Authors have been included in this manuscript based on the ICMJE recommendations. MS critically reviewed and supervised structuring of the manuscript and contributed to the development of the discussion. MTÁR designed the protocol, analysed, revised critically and gave final approval of the manuscript. GC critically reviewed and approved the manuscript. MJ critically reviewed and approved the manuscript. TM contributed to patient recruiting and collection of patient data. KM participated in discussions on the protocol, and reviewed the manuscript. J0 critically reviewed and approved the manuscript. SF critically reviewed and supervised structuring of the manuscript and contributed to the development of the discussion. MTR critically reviewed and approved the manuscript.

Funding This work was supported by Bayer.

Competing interests MS: Bayer employee. MTAR: Grants/research support: Shire/ Takeda. Company-sponsored speaker bureau: Shire/Takeda, Roche, Bayer, Amgen, Novartis, Novo Nordisk, Sobi, CSL Behring. GC: Grants/research support: Sobi, Pfizer, CSL Behring. Honoraria or consultation fees: Roche. Company-sponsored speaker bureau: Roche, CSL Behring, Sobi, Bayer, Novo Nordisk, uniQure, Kedrion, Shire. MJ: Consultant: Bayer, Takeda, Genentech, CSL Behring. Company-sponsored speaker bureau: Takeda. TM: Grants/research support: Bioverativ, KM Biologics. Honoraria or consultation fees: Shire, Chugai, Bayer, Novo Nordisk, CSL, Pfizer, uniQure, Bioverativ, KM Biologics. Honoraria or consultation fees: Shire, Chugai, Bayer, Novo Nordisk, CSL, Pfizer, Bioverativ. Grants/research support: Bayer, Sanquin, Pfizer. KM: Grants/research support: Bayer, Sanquin, Pfizer. Honoraria or consultation fees: uniQure. J0: Honoraria; fees for speaking, consulting or symposium/ congress attendance; or research funding: Bayer, Biogen Idec, Biotest, Chugai, CSL Behring, Grifols, Novo Nordisk, Octapharma, Pfizer, Roche, Shire, Swedish Orphan Biovitrum. SF: Bayer employee and shareholder. MTR: Grants/research support: Bayer, BioMarin. Honoraria or fees for advisory board/speaker bureau: Bayer, Novo Nordisk, Sanofi Genzyme, Takeda.

Patient consent for publication Not required.

Provenance and peer review Not commissioned; externally peer reviewed.

Supplemental material This content has been supplied by the author(s). It has not been vetted by BMJ Publishing Group Limited (BMJ) and may not have been peer-reviewed. Any opinions or recommendations discussed are solely those of the author(s) and are not endorsed by BMJ. BMJ disclaims all liability and responsibility arising from any reliance placed on the content. Where the content includes any translated material, BMJ does not warrant the accuracy and reliability of the translations (including but not limited to local regulations, clinical guidelines, terminology, drug names and drug dosages), and is not responsible for any error and/or omissions arising from translation and adaptation or otherwise.

Open access This is an open access article distributed in accordance with the Creative Commons Attribution Non Commercial (CC BY-NC 4.0) license, which permits others to distribute, remix, adapt, build upon this work non-commercially, and license their derivative works on different terms, provided the original work is properly cited, appropriate credit is given, any changes made indicated, and the use is non-commercial. See: http://creativecommons.org/licenses/by-nc/4.0/.

\section{ORCID iDs}

Karina Meijer http://orcid.org/0000-0001-9447-0465

M T Reding http://orcid.org/0000-0003-3319-2806

\section{REFERENCES}

1 Srivastava A, Brewer AK, Mauser-Bunschoten EP, et al. Guidelines for the management of hemophilia. Haemophilia 2013;19:e1-47.

2 Oldenburg J. Optimal treatment strategies for hemophilia: achievements and limitations of current prophylactic regimens. Blood 2015;125:2038-44.

3 Collins PW, Blanchette VS, Fischer K, et al. Break-through bleeding in relation to predicted factor VIII levels in patients receiving prophylactic treatment for severe hemophilia a. J Thromb Haemost 2009;7:413-20.

4 Thornburg CD, Duncan NA. Treatment adherence in hemophilia. Patient Prefer Adherence 2017;11:1677-86.

5 Reding MT, Ng HJ, Poulsen LH, et al. Safety and efficacy of Bay 94-9027, a prolonged-half-life factor VIII. J Thromb Haemost 2017;15:411-9.

6 Mei B, Pan C, Jiang $\mathrm{H}$, et al. Rational design of a fully active, long-acting PEGylated factor VIII for hemophilia a treatment. Blood 2010;116:270-9. 
7 Coyle TE, Reding MT, Lin JC, et al. Phase I study of BAY 94-9027, a PEGylated B-domain-deleted recombinant factor VIII with an extended half-life, in subjects with hemophilia A. J Thromb Haemost 2014:12:488-96.

8 Shah A, Coyle T, Lalezari S, et al. BAY 94-9027, a PEGylated recombinant factor VIII, exhibits a prolonged half-life and higher area under the curve in patients with severe haemophilia A: comprehensive pharmacokinetic assessment from clinical studies. Haemophilia 2018;24:733-40.

9 Shah A, Solms A, Wiegmann S, et al. Direct comparison of two extended-half-life recombinant FVIII products: a randomized, crossover pharmacokinetic study in patients with severe hemophilia A. Ann Hematol 2019;98:2035-44.

10 Solms A, Shah A, Berntorp E, et al. Direct comparison of two extended half-life PEGylated recombinant FVIII products: a randomized, crossover pharmacokinetic study in patients with severe hemophilia A. Ann Hematol 2020;99:2689-98.

11 Santagostino E, Lalezari S, Reding MT, et al. Safety and efficacy of Bay 94-9027, an extended-half-life factor VIII, during surgery in patients with severe hemophilia A: results of the protect VIII clinical trial. Thromb Res 2019;183:13-19.

12 Lalezari S, Reding MT, Pabinger I, et al. BAY 94-9027 prophylaxis is efficacious and well tolerated for up to $>5$ years with extended dosing intervals: PROTECT VIII extension interim results. Haemophilia 2019;25:1011-9.

13 Dendere R, Slade C, Burton-Jones A, et al. Patient portals facilitating engagement with inpatient electronic medical records: a systematic review. J Med Internet Res 2019;21:e12779.

14 Lau M, Campbell H, Tang T, et al. Impact of patient use of an online patient portal on diabetes outcomes. Can J Diabetes 2014;38:17-21.

15 Mancuso ME, Reding MT, Negrier C, et al. Decreased bleeding rates in patients with hemophilia a switching from standardhalf-life FVIII to Bay 94-9027 prophylaxis. Thromb Haemost 2021;121:1079-86.

16 Aledort L, Milligan S, Watt M, et al. A retrospective observational study of Rurioctocog alfa pegol in clinical practice in the United States. J Manag Care Spec Pharm 2020;26:492-503. 